\title{
Endoscopic Treatment for Obesity: New Emerging Technology Trends
}

\author{
Seung Han Kim and Hoon Jai Chun
}

Division of Gastroenterology and Hepatology, Department of Internal Medicine, Institute of Gastrointestinal Medical Instrument Research, Korea University College of Medicine, Seoul, Korea

Obesity is a complicated metabolic disease and can be defined as a condition in which excess body fat has been stored in such an amount that health may be negatively affected. ${ }^{1}$ It is responsible for around 5\% of all global deaths and strongly associated with the metabolic syndrome, which has increased significantly in recent decades. In 2014, the McKinsey Global Institute reported that the economic impact of obesity is roughly $2.8 \%$ of global domestic product, or $\$ 2.0$ trillion, approximately equivalent to the worldwide impact of armed violence or smoking, terrorism, and war. The global economic effect of obesity is increasing, and no country has yet reversed its obesity epidemic. ${ }^{2}$ If recent trends in the prevalence of obesity continue, by 2030 almost half of the world's adult population could be obese or overweight. ${ }^{3}$

Obesity is the consequence of multifactorial causes, and no single solution can be effective in reversing it. Current common treatment strategies for obesity include lifestyle modification, diet, pharmacologic therapy, and bariatric surgery. However, lifestyle modification and use of pharmacological agents have high attrition rates, and limited effectiveness, and durability. ${ }^{4}$ Surgical interventions, such as sleeve resection or gastric bypass, are the most effective treatments to date, but are currently indicated only for the very obese (body mass index [BMI] greater than $40 \mathrm{~kg} / \mathrm{m}^{2}$, or BMI 35 to $40 \mathrm{~kg} / \mathrm{m}^{2}$ with an obesity-related disorder). Bariatric surgery is associated with both a significant decrease in overall mortality and long-term weight loss; however, the majority will not be candidates for bariatric surgery, and even if indicated, the decision to undergo a surgical procedure can be difficult. There is still a significant complication rate and a small, but real perioperative mortality rate. ${ }^{5}$

The endoscopic approach may have the potential for less invasive intervention in obesity treatment, with appropriate weight loss, reduced morbidity, and easy access for obese patients. As minimally invasive therapy, endoscopic bariatric treatment can have several advantages over current therapies.
It can be performed through a simple endoscopic procedure, and can be reversible through use of a intragastric balloon, suture technique, endoluminal malabsorptive device, or aspiration therapy. The various endoscopic bariatric procedures differ in their mechanisms of effect, and have various degrees of strength, persistence, and reversibility. Therefore, endoscopic bariatric therapy could play a diverse role in obesity treatment according to the degree of obesity, and evaluation for its use can be based on the overall risk-benefit ratio.

Intragastric balloons and suturing methods employ spaceoccupying and volume restriction mechanisms. Intragastric balloons were designed to be retained for 6 months within the stomach before their elimination, and balloon placement was associated with a significantly reduced food intake in obese patients. ${ }^{6}$ Suturing techniques, such as endoluminal vertical gastroplasty, transoral gastroplasty, and a transoral endoscopic restrictive implant system, were developed as endoscopic skill evolved, ${ }^{7}$ and showed good outcomes in preliminary research. ${ }^{8}$ With these methods, there were no serious adverse events; however, body weight was regained following balloon removal, and inadequate durability of sutures should be considered. ${ }^{8}$ In addition, suturing methods are problematic for gastric cancer screening after the procedure, require high endoscopic skills and time, and are still in the early stages of development.

The malabsorption technique is designed to inhibit nutrient contact with the mucosa of the proximal intestine, in addition to delaying gastric emptying and causing early satiety. Although there is a risk of device migration, obstruction, and persistent abdominal pain, the endoluminal malabsorptive device was mostly safe when implanted, and resulted in significant weight reduction and improvement in cardiometabolic parameters. ${ }^{8}$

Novel methods are introduced continuously. One of these uses percutaneous endoscopic gastrostomy tube technology by aspirating a portion of an ingested meal from the stomach; this removed up to approximately $30 \%$ of the calories ingested in a

Correspondence to: Hoon Jai Chun

Division of Gastroenterology and Hepatology, Department of Internal Medicine, Institute of Gastrointestinal Medical Instrument Research, Korea

University College of Medicine, 73 Inchon-ro, Seongbuk-gu, Seoul 136-705, Korea

Tel: +82-2-920-6555, Fax: +82-2-953-1943, E-mail: drchunhj@chol.com

pISSN 1976-2283 eISSN 2005-1212 http://dx.doi.org/10.5009/gnl15125

@ This is an Open Access article distributed under the terms of the Creative Commons Attribution Non-Commercial License (http://creativecommons.org/licenses/by-nc/4.0) which permits unrestricted non-commercial use, distribution, and reproduction in any medium, provided the original work is properly cited. 
meal, and appeared to offer a safe and viable long-term weight reduction treatment. ${ }^{9}$

Recently, the Food and Drug Administration (FDA) approved an implantable weight reduction device, called the Maestro system, which uses electrical impulses to control appetite. It mimics a heart pacemaker and is currently available for implantation by a surgeon. Although it is only approved for people who are severely overweight, the FDA concluded that the benefits outweighed the risks, and at some point endoscopic deployment is expected.

A safe, durable, and effective endoscopic treatment method for obesity is clearly needed. However, there are considerable limitations to endoscopic bariatric treatment, such as inadequate durability, threshold for treatment, or lack of established parameters for the procedures.

In addition, except for the intragastric balloon, the majority of the other endoscopic bariatric treatment methods are still in their early stages, as evidenced by the small number of clinical trials found in the literature. However, advances in endoscopic bariatric devices now permit the possibility of exploring bariatric therapy with flexible endoscopy.

For the management of obese patients, a multidisciplinary approach is crucial for effective care. With the approval of novel antiobesity drugs by the U.S. FDA in 2012, such as a phentermine-topiramate combination and lorcaserin, traditional obesity treatments are continuously improving. Accompanied by these trends, emerging endoscopic technology may have a significant role, by providing superior efficiency to pharmacological therapy, with lower risk than conventional bariatric surgery.

The endoscopic approach to obesity can be promising for intervention in patients with varying degrees of obesity, and has the potential to provide a more cost-effective outpatient procedure for the increasing population of people with obesity and the metabolic syndrome. Trained gastroenterologists have an opportunity to affect this significant epidemic. We may need to be more active in establishing standards and defining satisfactory outcomes, become more interested in the treatment of obese patients, and involved in the development of endoscopic bariatric treatment for the reversal of the obesity epidemic.

\section{CONFLICTS OF INTEREST}

No potential conflict of interest relevant to this article was reported.

\section{ACKNOWLEDGEMENTS}

This work was supported by the National Research Foundation of Korea (NRF) grant funded by the Korean Government (NRF-2014R1A2A2A01006131) and Grant of Korea University.

\section{REFERENCES}

1. Obesity: preventing and managing the global epidemic. Report of a WHO consultation. World Health Organ Tech Rep Ser 2000;894: i-xii, 1-253.

2. Kleinert S, Horton R. Rethinking and reframing obesity. Lancet. Epub 2015 Feb 18. http://dx.doi.org/10.1016/S0140-6736(15)60163-5.

3. Kelly T, Yang W, Chen CS, Reynolds K, He J. Global burden of obesity in 2005 and projections to 2030. Int J Obes (Lond) 2008; 32:1431-1437.

4. Kakkar AK, Dahiya N. Drug treatment of obesity: current status and future prospects. Eur J Intern Med 2015;26:89-94.

5. Sharma A, Aggarwal S, Ahuja V, Bal C. Evaluation of gastroesophageal reflux before and after sleeve gastrectomy using symptom scoring, scintigraphy, and endoscopy. Surg Obes Relat Dis 2014;10:600-605.

6. Kentish SJ, Page AJ. The role of gastrointestinal vagal afferent fibres in obesity. J Physiol 2015;593:775-786.

7. Song Y, Choi HS, Kim K, et al. A simple novel endoscopic successive suture device: a validation study for closure strength and reproducibility. Endoscopy 2013;45:655-660.

8. Mathus-Vliegen EM. Endoscopic treatment: the past, the present and the future. Best Pract Res Clin Gastroenterol 2014;28:685-702.

9. Sullivan S, Stein R, Jonnalagadda S, Mullady D, Edmundowicz S. Aspiration therapy leads to weight loss in obese subjects: a pilot study. Gastroenterology 2013;145:1245-1252.e5. 